\title{
CM-P00056857
}

\section{RESONANCE AND DIRECT GAMMA RAY INTERACTIONS}

\author{
I. Estrada
}

University of Mexico

and

H. Feshbach *)

\section{A B S T R A C T}

The results of the projection operator formalism for nuclear reactions are evaluated for gamma ray processes. A direct term and a resonance term in the Breit-Wigner form are obtained. The usual expression for the gamma ray width is found to be incorrect. One must also include the process in which the compound nucleus decays by particle emission but the latter radiates on the "way out" via the direct gamma ray interaction. The analogue of the Ward idendity for many body systems is derived.

*) CERN Ford Foundation Fellow. On leave from Massachusetts Institute of Technology, Cambridge, Mass., U.S.A. 
A. INTRODUCTION

In presenting this paper we have two purposes in mind. In the paper "Unified Theory of Nuclear Reactions II" 1)*) it was asserted that the emission or absorption of gamma rays could be treated on exactly the same footing as the emission or absorption of heavy particles. We intend to justify this remark and thus show how gamma ray processes fit naturally into the reaction formalism developed in II. This formalism includes explicitly the so-called direct interaction mechanism so that our discussion will lead to a formalism for the direct interaction between gamma rays and nuclei. The direct interaction will also affect the expression for the gamma ray widths while the existence of the compound nucleus will modify the direct interaction.

Some of these matters have been discussed earlier. Lane and Thomas 3) have extended the Wigner $R$ matrix formalism so as to include gamma ray interactions. Direct interaction of gamma rays has been studied by many authors including R. Christy 4), Lane and Lynn ${ }^{5)}$ and Guth, Francis and Goldman 6). C. Shakin 7) has used a particular realization of the formalism of II but his derivation is valid only when the radiation width is small compared to the particle width.

*) To be referred to as II. An earlier paper ${ }^{2)}$ will be referred to as I. 
B.

We start with the following formulae derived in II valid in the region in which the compound nuclear resonances are isolated. The transition amplitude $\mathcal{J}$ for a given reaction $\alpha \rightarrow \beta$ may be written as the sum of two amplitudes; the direct and the resonant, viz. :

$$
J(\beta \mid x)=J_{p}(\beta \mid \alpha)+J_{R}(\beta \mid \alpha)
$$

Here $J_{R}$ has the Breit-Wigner form

$$
J_{R}(\beta \mid \alpha)=\frac{\left\langle\psi_{\beta}^{(-)} H_{P Q} \Phi_{S}\right\rangle\left\langle\Phi_{S} H_{Q P} \psi_{\alpha}^{(t)}\right\rangle}{E-E_{S}+i \pi\left\langle\Phi_{S} H_{Q P} \delta\left(E-H^{\prime}\right) H_{P Q} \Phi_{S}\right\rangle}
$$

where. $H$ is the Hamiltonian of the entire system, $P$ is a projection operator on the open channel sub-space discussed in II, $Q \equiv(1-P)$ and

$$
H_{P Q}=P H Q \quad H_{Q Q}=Q H Q \text { etc. }
$$

The function $\Phi_{s}$ is a normalized bound state eigenfunction of the operator $\mathrm{H}_{Q Q}$ :

$$
H_{Q Q} \Phi_{S}=\varepsilon_{s} \Phi_{S}
$$

$\mathrm{E}_{\mathrm{S}}$ is shifted from $E_{\mathrm{s}}$ :

$$
E_{s}=\varepsilon_{s}+\left\langle\Phi_{s} H_{Q P} \frac{\rho}{E^{+}-H^{\prime}} H_{P Q} \Phi_{s}\right\rangle
$$


We may define a projection operator for $\Phi_{\mathrm{s}}$ as follows

$$
\left.p_{s} \equiv \Phi_{s}\right\rangle\left\langle\Phi_{s}\right.
$$

and

$$
q_{s} \equiv 1-p_{s}
$$

Then $H^{\prime}$ is given by

$$
H^{\prime}=H_{P P}+H_{P Q} \frac{q_{s}}{E-H_{Q Q}} H_{Q P}
$$

The functions $\psi_{\alpha}{ }^{(+)}$and $\psi_{\beta}{ }^{(-)}$are solutions of the Schroedinger equation

$$
H^{\prime} \psi=E \psi
$$

The superscripts $(+)$ and $(-)$ denoting as usual diverging and converging solutions while the subscripts $(\alpha)$ and $(\beta)$ refer to possible incident plane wave (or if either necessary or convenient distorted incident waves). Finally, $J_{p}$ is the transition amplitude which follows from Eq. (B.6). We shall not repeat our discussion of these formulae as given in II. We need only mention here that we have separated the amplitude into two terms, one of which varies slowly with the energy and which, therefore, is referred to as direct or potential interaction while the second varies rapidly with $E$ and is the compound nucleus term. 
To apply (B.1) to a particular case it is only necessary to specify $\mathrm{H}$, the Hamiltonian and, P, the projection operator on the open channel subspace. For our present problem, it is convenient to break $H$ up into the usual three parts :

i) the heavy particle Hamiltonian, $\mathrm{H}^{\mathrm{N}}$, which describes the interaction in the absence of an electromagnetic field,

ii) $H^{\gamma}$, the Hamiltonian for the free radiation field, and

iii) $H^{\gamma}$, that part of the Hamiltonian describing the interaction between the nucleons and radiation.

We shall approximate $\mathrm{H}^{\mathbb{N}}$ by its "single photon" part so that

$$
H^{\gamma N} \simeq-\frac{e}{c} \sum_{i} j_{i} \cdot A_{m}
$$

where $j_{i}$ is the current density for the $i^{\prime}$ th proton and $A_{i}$ the vector potential.

Consistent with this approximation we shall consider only the open channel zero photon and one photon states. Further only a single one photon state is required; namely that one which describes the particular photon of energy to tiw whose emission or absorption is being considered. The corresponding open channel projection operator is then :

$$
P=P_{0}^{(N)} p_{0}^{(\gamma)}+P_{1}^{(N)} P_{1}^{(\gamma)} \equiv P_{0}+P_{1}
$$

where $\mathrm{P}_{0}^{(N)}$ is the heavy particle projection operator which projects out those residual nuclear states which are possible when there are no photons emitted (or absorbed) while $\mathrm{P}_{1}^{(\mathrm{N})}$ projects out the possible residual nuclear states associated with the emission (or absorption) of a photon of a given frequency. 
These heavy particle operators have been discussed in II. The operators

and $p_{1}(\gamma)$ project out the zero photon state vector and the one photon state vector describing a photon of frequency $\omega$ respectively. Hence

$$
p_{0}^{(\gamma)} p_{1}^{(\gamma)}=0
$$

From the restriction to zero and one photon states it follows that

$$
Q=Q_{0}^{(N)} p_{0}^{(\gamma)}+Q_{1}^{(N)} p_{1}^{(\gamma)} \equiv Q_{0}+Q_{1}
$$

We have now completed the definitions of the various symbols which occur in (B.1) and (B.2). These equations and the associated definitions provide the kinematical framework needed for the discussion of gamma ray resonance and direct reactions. However, it would be most useful and as we shall see illuminating to re-express some of the quantities, particularly the matrix elements in $J_{R}$ and $J_{p}$ in more familiar terms. The absence of an immediate correspondence stems from the fact that $P$ and $Q$ involve both heavy particles and the photon and it is thus important to disentangle these two aspects as much as possible. However, as we shall see, this operation can only be made approximately because of direct gamma ray interaction effects so that the customary formulae for widths, for example, hold exactly only in their absence.

Our first step in this programme is to make the zero and one photon parts of the interactions and wave functions $\psi_{\alpha}{ }^{(+)}, \psi_{\beta}(-)$ and $\Phi_{s}$ explicit. Let us consider the function $\psi$ first and let the zero photon part of $\psi, \mathrm{P}_{0} \psi$ be $\psi(0)$ while the one photon part, $\mathrm{P}_{1} \psi$, is denoted by $\psi(1)$. Finally, note that since $H^{\prime}$ operates only on open channel wave functions that

$$
H^{\prime}=P_{0} H^{\prime} P_{0}+P_{1} H^{\prime} P_{1}+P_{0} H^{\prime} P_{1}+P_{1} H^{\prime} P_{0}
$$


We then obtain the following equations for $\psi(0)$ and $\psi(1)$ :

$$
\begin{aligned}
& \left(E-H_{N}^{\prime}\right) \psi(0)=\left(P_{0} H^{\prime} P_{1}\right) \psi(1) \\
& \left(E-\hbar \omega-H_{N}^{\prime}\right) \psi(1)=\left(P_{1} H^{\prime} P_{0}\right) \psi(0)
\end{aligned}
$$

Here $H_{N}^{\prime}$ is just the operator $H^{\prime}$ in the absence of electromagnetic interaction, i.e.,

$$
H_{N}^{\prime}=H^{\prime}(e=0)
$$

This pair of coupled equations gives a stationary state formulation of the direct interaction absorption and emission of radiation. Note that in the absence of the radiation terms on the right hand side of (B.11), the equations for $\psi(0)$ and $\Psi(1)$ are simply the direct interaction Schroedinger equations with available energy $E$ and $(E-\hbar \omega)$ respectively. The radiative terms couple these two states but because the interaction is relatively weak we can still classify the solutions of (B.11) as being either principally one photon (available energy for heavy particles $E-\hbar \omega)$ or zero photon states respectively. We shall indicate which solution we are considering by a subscript. The coupling terms $\left(\mathrm{P}_{0} \mathrm{H}^{\prime} \mathrm{P}_{1}+\mathrm{P}_{1} \mathrm{H}^{\prime} \mathrm{P}_{0}\right)$ contain not only the usual $\mathrm{H}^{\gamma \mathrm{N}}$ but also terms arising from $\left.\mathrm{H}_{\mathrm{PQ}} \sqrt{\mathrm{q}}_{\mathrm{S}} /\left(\mathrm{E}-\mathrm{H}_{\mathrm{QQ}}\right)\right]_{\mathrm{H}}$. These interaction terms as we shall call them are a consequence of the momentum dependence, (or in other words the nonlocality) of the effective Hamiltonian for direct interactions and potential scattering. We shall discuss the interaction terms more explicitly in Section C., but for the present we shall need only their formal representation. 
The transition matrix for direct interaction gamma ray processes can be readily obtained from (B.11). We find :

$$
J_{p}(\beta \mid \alpha)=\left\langle\psi_{\beta 1}^{(-)}(1) P_{1} H^{\prime} P_{0} \psi_{\alpha, 0}^{(+)}(0)\right\rangle
$$

The function $\psi_{\alpha_{0}^{(+)}}^{(0)}$ is a solution the homogeneous form of (B.11a), $\psi_{\beta 1}^{(-)}(1)$ of $(\mathrm{B} .11 \mathrm{~b})$.

Equations similar to $\left(B_{.11}\right)$ can be written for the zero and one photon parts of $\Phi_{S}$. Denoting these by $\overline{1}_{s}(0)$ and $\Phi_{s}(1)$ respectively we obtain

$$
\begin{aligned}
& \left(\varepsilon_{S}-H_{Q Q}^{(N)}\right) \Phi_{S}(0)=H_{Q Q}^{(\gamma N)} \Phi_{S}(1) \\
& \left(\varepsilon_{S}-\hbar \omega-H_{Q Q}^{(N)}\right) \Phi_{S}(1)=H_{Q Q}^{(\gamma N)} \Phi_{S}(0)
\end{aligned}
$$

We have omitted the $P_{i}$ operations in these equations since their presence is indicated by the nature of the functions upon which the $\mathrm{H}^{(\mathbb{N})}$ and $\mathrm{H}^{\left(\gamma_{N}\right)}$ operate. Note again that there are two sets of eigenvalues, $\mathcal{U}_{S}$, for this pair of coupled equations corresponding to states whose principal component is a zero or one photon state. The mixing of these corresponds to the possibility of these states absorbing or emitting a single photon of energy $\hbar \omega$. The energy of the "zero photon" state is approximately, $\mathcal{E}_{\mathrm{s}}^{(\mathrm{N})}$, the wave function $\Phi_{\mathrm{s}}^{(\mathrm{N})}$, the energy and wave function in the absence of radiation. To the same approximation the one photon state is also described by the wave function $\Phi_{\mathrm{S}}(\mathrm{N})$ but its energy is, of course, $E_{\mathrm{s}}^{(\mathrm{N})}+\hbar \omega$.

Finally, we can write down the matrix elements in Eq. (B.2) in a form in which the electromagnetic terms of $\mathrm{H}_{Q \mathrm{P}}$ are explicitly given. For

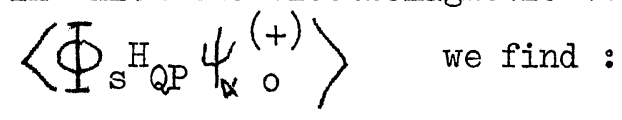


8.

$$
\begin{aligned}
\left\langle\Phi_{S} H_{Q P} \Psi_{\alpha}^{(+)}\right\rangle & =\left\langle\Phi_{S}(0) H_{Q P}^{N} \psi_{\alpha}^{(+)}(0)\right\rangle+\left\langle\Phi_{S}^{(0)} H_{Q P}^{\gamma N} \psi_{\alpha}^{(t)}(1)\right\rangle \\
& +\left\langle\Phi_{S}(1) H_{Q P}^{N} \Psi_{\alpha}^{(+)}(1)\right\rangle+\left\langle\Phi_{s}(1) H_{Q P}^{\gamma N} \psi_{\alpha}^{+}(0)\right\rangle
\end{aligned}
$$

Similarly

$$
\begin{aligned}
\left\langle\psi_{\beta}^{(-)} H_{P Q} \Phi_{S}\right\rangle & =\left\langle\psi_{\beta}^{(0)} H_{P Q}^{N} \Phi_{S}(0)\right\rangle+\left\langle\psi_{\beta}^{(-)}(1) H_{P Q}^{\gamma N} \Phi_{S}(0)\right\rangle \\
& +\left\langle\psi_{\beta}^{(-)}(1) H_{P Q}^{N} \Phi_{S}(1)\right\rangle+\left\langle\psi_{\beta}^{(-)}(0) H_{P Q}^{\gamma N} \Phi_{S}(1)\right\rangle
\end{aligned}
$$

With these equations (B.11), (B.13), (B.14) and (B.15) it becomes possible to extract the major dependence on the electromagnetic coupling for the product of the matrix elements for the possible channels as they occur in the numerator of the resonance term, (B.2).

i) Consider first the non-radiative channels. In this case $\psi_{(\alpha, \beta}( \pm)$ are of zero order while $\psi_{\alpha, \beta}{ }^{( \pm)}$(1) are of first order in the electromagnetic coupling. For the wave function $\Phi s$, we take that solution of (B.13) for which $\Phi_{S}(0)$ is of zero order, $\Phi_{S}(1)$ of first order. The other solution leads to second order corrections. For these $\psi$ and $\Phi$ functions we find

$$
\begin{aligned}
\left\langle\psi_{\beta}^{(-)} H_{P Q} \Phi_{S}\right\rangle\left\langle\Phi_{S} H_{Q P} \psi_{\alpha}^{(+)}\right\rangle & \\
= & \left\langle\psi_{\beta}^{(-1}(0) H_{P Q}^{N} \Phi_{S}(0)\right\rangle\left\langle\Phi_{S}(0) H_{Q P}^{N} \psi_{\alpha}^{(+)}\right\rangle \\
& + \text {terms of second order }
\end{aligned}
$$

This is just the result which prevails in the absence of radiation so that the expression for particle width ${ }^{1), 2)}$ is not modified by the electromagnetic interaction. 
ii) We consider next the radiation channel in which a photon is emitted. For this channel, the zero photon part of the incident wave function, $\psi_{\alpha}{ }^{(+)}(0)$, is of zero order, while $\psi_{\alpha}^{(+)}(1)$ is of first order. The situation is reversed for the emergent channel wave function for $\psi_{\beta}^{(-)}(1)$ is now of zero order while $\psi_{\beta}^{(-)}(0)$ is of first order. Turning to the compound nucleus state $\Phi_{\mathrm{s}}$ we shall first consider the most interesting physical case in which $\Phi_{S}(0)$ is the principal term while $\Phi_{s}(1)$ is of first order. We then find that

$$
\left\langle\Phi_{S} H_{Q P} \psi_{\alpha}^{(+)}\right\rangle=\left\langle\Phi_{s}(0) H_{\Gamma_{Q}}^{N} \psi_{\alpha}^{(t)}(0)\right\rangle_{+} \text {terms of second order }
$$

while

$$
\begin{aligned}
\left\langle\psi_{\beta}^{(-)} H_{P Q} \Phi_{S}\right\rangle & =\left\langle\psi_{\beta 1}^{(-)}(0) H_{P Q}^{N} \Phi_{S O}(0)\right\rangle+\left\langle\psi_{\beta 1}^{(-)}(1) H_{P Q}^{O N} \Phi_{S O}(0)\right\rangle \\
& +\left\langle\psi_{\beta(}^{(-)}(1) H_{P Q}^{N} \Phi_{S O}(1)\right\rangle
\end{aligned}
$$

$$
+ \text { terms of second order }
$$

In this last equation we need to make the first order character of the first and third term explicit by substituting for $\psi_{p i}^{(-)}(0)$ from Eq. (B.11) and for $\Phi_{\text {so }}(1)$ from (B.13). We obtain

$$
\begin{aligned}
\left\langle\psi_{\beta}^{(-)} H_{P Q} \Phi_{S}\right\rangle & =\left\langle\psi _ { \beta } ^ { ( - ) } ( 1 ) \left\{ H_{P Q}^{\gamma N}+H_{P Q}^{N} \frac{1}{E_{S}-\hbar \omega-H_{Q Q}^{N}}{ }_{(B .19)}^{H_{Q}}\right.\right. \\
& \left.\left.+P_{1} H^{\prime} P_{0} \frac{1}{E^{+}-H_{N}^{\prime}} H_{P Q}^{N}\right\} \Phi_{I_{S}}(0)\right\rangle
\end{aligned}
$$


An important simplification in this result is possible as a consequence of the relation between the full wave function $\Psi_{\beta}{ }^{(-)}$describing the final state and its projection on the open channel $\psi_{\beta}(-)$ :

$$
\Psi_{\beta}^{(-)}=\left[1+\frac{1}{E-\hbar \omega-H_{Q Q}^{N}} H_{Q P}\right] \psi_{\beta}^{(-)}(1)
$$

Disregarding the small difference between $E$ and $\mathcal{E}_{\mathrm{s}}$ (since we are dealing with the resonant term) (B.19) becomes:

$$
\begin{aligned}
\left\langle\psi_{\beta}^{(-)} H_{P Q} \Phi_{S}\right\rangle= & \left\langle\Psi_{\beta}^{(-)}(1) H^{\gamma N} \Phi_{s 0}(0)\right\rangle \\
& +\left\langle\Psi_{\beta}^{(-)}(1) P_{1} H^{\prime} P_{0} \frac{1}{E^{+}-H_{N}^{\prime}} H_{P Q}^{N} \Phi_{S O}(0)\right\rangle
\end{aligned}
$$

The first of these terms is the familiar form which is commonly employed in the calculation of radiation widths. The second is an important correction arising from a process in which the compound nucleus decays by particle emission via $H_{P Q}^{N}$ but the system on the "way out" according to $\left(1 / \mathrm{E}^{+}-\mathrm{H}_{\mathrm{N}}^{\prime}\right)$ radiates via $\left(\mathrm{P}_{1} \mathrm{H}^{\prime} \mathrm{P}_{0}\right)$. If we take the contribution of the $\delta$ function part of the propagator in the second term we obtain a contribution to the width arising from radiation in the wave zone only. That this term must exist was first emphasized by N. Francis ${ }^{8)}$ and has been the major subject treated by C. Shakin 7). The existence of this second mode of decay, if it is sufficiently uncorrelated with the familiar first term of (B.21) may have an important effect on the statistics of the gamma ray widths. This conjecture needs further investigation.

We turn next to the case where the compound state $\Phi_{\mathrm{s}}$ contains a single photon; i.e., the zero order component is $\Phi_{s}(1)$. As can be verified by following through the considerations just above (in the present case the zero order wave functions are $\psi_{\alpha_{0}}^{+}(0), \psi_{\beta 1}^{(-)}(1)$ and $\Phi_{s 1}(1)$; 
the first order $\left.\psi_{\alpha_{0}}^{+}(1), \psi_{\beta_{1}}{ }^{(-)}(0), \Phi_{s 1}(0)\right)$ this case would require. that the compound nucleus be formed upon the emission of the photon. Clearly a resonance can occur only if an eigenvalue of $\mathrm{H}_{Q Q}^{\mathrm{N}}$ falls near $E-\hbar \omega$, the energy available to the nuclear system after the emission. Thus this type of resonance can only occur for $\left(n, n^{\prime} \gamma\right)$ reactions and will not contribute to the $(n, \gamma)$ reaction under discussion here. With regard to the $\left(n, n^{\prime} \gamma\right)$ reaction it is clear that the amplitude for this resonance process in which the compound nucleus is formed after photon emission is equal in importance to the processes discussed earlier in which the radiation is emitted after the formation of the compound nucleus. The results which apply when the emitted photon is in the $d \omega / \omega$ part of the spectrum have been discussed elsewhere ${ }^{9)}$. 
The effective interaction through which the direct gamma ray processes proceed, $\mathrm{P}_{1} \mathrm{H}^{\prime} \mathrm{P}_{0}$, has only been given in this symbolic form. It is possible to be somewhat more explicit by inserting (B.5) for $\mathrm{H}^{\prime}$ and extracting the first order electromagnetic terms. However, no useful purpose is served by exhibiting these leng:thy formulae unless a specific form of the nuclear projection operators is used and the indicated calculations performed.

In this section, we examine the question as to how much information on the electromagnetic interaction can be obtained from the momentum dependence, i.e., the non-local nature of $\mathrm{H}_{\mathrm{N}}$. It has often been suggested that this momentum dependence implies an additional electromagnetic interaction which could be obtained via the substitution $\underset{d}{p} \rightarrow p-\mathrm{e} / \mathrm{c}$. An equivalent remark points out that the velocity operator, computed as the commutator of $\underline{r}$ and the effective Hamiltonian, differs from $\mathrm{p} / \mathrm{m}$ when the Hamiltonian is momentum dependent and, therefore, the electromagnetic interaction term $\mathrm{V} \cdot \mathrm{A}$ will have a modified momentum dependence. Both of these points of view lead to the suggestion that one should take the current vector associated with a proton as $\partial \mathrm{H}_{\mathrm{N}} / \partial \mathrm{p}$ where $\mathrm{p}$ is the momentum operator for the proton.

We shall now look into this question limiting the applicability of our considerations by the following assumptions. First we assume that our particles are spinless so that the complete electromagnetic interaction is given by replacing $\frac{p_{i}}{m}$ by $p_{i}-e / c A_{i}$ in the Hamiltonian $H$ describing the complete system. Secondly we shall consider the full effective Hamiltonian so that resonance terms are included.

$$
H_{e f f}=H_{P P}+H_{P Q} \frac{1}{E-H_{Q Q}} H_{Q P}
$$

This will describe direct interactions when the energy $E$ is between widely spaced resonances. The electromagnetic interaction following from (c.1) is : 


$$
\begin{aligned}
& \left(H_{e f f}\right)_{\gamma N}=\sum_{i}\left\{P\left(\frac{\partial H^{N}}{\partial p_{i}} \cdot A_{i}\right) P+P\left(\frac{\partial H^{N}}{\partial p_{i}} \cdot A_{i}\right) Q \frac{1}{E-H_{Q Q}^{N}}\left(H^{N}\right)_{Q P}\right. \\
& +H_{P Q}^{N} \frac{1}{E-H_{Q Q}^{N}} Q\left(\frac{\partial H^{N}}{\partial P_{i}} \cdot A_{i}\right) P \\
& \left.+H_{P Q}^{N} \frac{1}{E-H_{Q Q}^{N}} Q \frac{\partial H^{N}}{\partial P_{i}} \cdot A_{i} Q \frac{1}{E-H_{Q Q}^{N}} H_{Q P}^{N}\right\}
\end{aligned}
$$

We now compare this with the results obtained by differentiating $\left(H_{e f f}^{\mathbb{N}}\right)$ with respect to one of the momentum operators, say $\underset{m}{p}$.

$$
P \frac{\partial H_{e f f}^{N}}{\partial p} P=P \frac{\partial}{\partial p}\left(P H^{N} P+P H^{N} Q \frac{1}{E-Q H^{N} Q} Q H^{N} P\right) P \text { (c.3) }
$$

Note, and this is most important, that $\partial / \hat{\partial} \underset{\sim}{p}$ operates on the projection operator $P$ and $Q$ as well as in $H^{N}$. In this connection, the following results are useful :

$$
\begin{aligned}
& P \frac{\partial P}{\partial P}=\frac{\partial P}{\partial P} Q \\
& \frac{\partial P}{\partial P} P=Q \frac{\partial P}{\partial P} \\
& \frac{\partial Q}{\partial P} P=Q \frac{\partial Q}{\partial P} \\
& \frac{\partial Q}{\partial P}=\frac{\partial Q}{\partial P} Q
\end{aligned}
$$

and finally that

$$
\frac{\partial P}{\partial p}=-\frac{\partial Q}{\partial P_{m}}
$$


With the aid of these rules it is an easy matter to calculate the right hand side of (C.3). We obtain

$$
\begin{aligned}
P \frac{\partial H_{e f f}^{N}}{\partial P} P & =\frac{\partial H^{N}}{\partial P} P+P \frac{\partial H^{N}}{\partial P} Q \frac{1}{E-H_{Q Q}^{N}} H_{Q P}^{N}+H_{P Q}^{N} \frac{1}{E-H_{Q Q}^{N}} \frac{\partial H^{N}}{\partial P} P \\
& +H_{P Q}^{N} \frac{1}{E-H_{Q Q}^{N}} Q \frac{\partial H^{N}}{\partial P} Q \frac{1}{E-H_{Q Q}^{N}} H_{Q P}^{N} \\
& +\left[E-\left(H^{N}\right)^{\prime}\right] \frac{\partial P}{\partial P} \frac{1}{E-H_{Q Q}^{N}} H_{Q P}^{N}+H_{P Q}^{N} \frac{1}{E-H_{Q Q}^{N}} \frac{\partial P}{\partial P}\left[E-\left(H^{N}\right)^{\prime}\right]
\end{aligned}
$$

Comparing (c.6) and (c.2) we see that for the matrix element of the gamma ray interaction we can take the current vector $J$ for the system as $\sum \partial H_{\text {eff }}^{N} \partial p_{i}$ in the limit of $\underline{\omega}$, the photon momentum and energy going: to zero. The restriction to the limiting value of zero photon momentum is a consequence of the possibility of radiative processes occurring while the incident particle and the target nucleus form a compound system, as is indicated by the fourth term on the right hand side of (c.2). Our result is then

$$
\underset{m \rightarrow}{J} \frac{\sum_{i}}{\partial H_{e f f}^{N}} / \partial p_{i} \text { as } \underset{m}{\omega} \rightarrow 0
$$

the sum being taken over the protons only. Equation (C.7) is the analogue for many body systems of the ward identity in quantum electrodynamics ${ }^{10)}$. It can be employed to obtain the effective nuclear charge as a function of $\underset{\mathrm{m}}{\mathrm{p}}$ but this would be valid only in the limit $\omega_{m} \rightarrow 0$. 
The condition $\omega \rightarrow 0$ implies that (C.7) is applicable only to electric dipole transitions. Equivalent results have been emploved in the calculation of the cross-section for long wavelength bremsstrahlung associated with a reaction 9),11). The cross-section for this process can be expressed. completely in terms of the reaction amplitudes in the absence of radiation and is otherwise insensitive to the details of the nucleon-nucleus interaction. For electric dipole transitions involving bound states it is possible to replice $\partial \mathrm{H}_{\mathrm{N}} / \partial \underset{\mathrm{m}}{\mathrm{p}}$ by the commutator of $\mathrm{H}_{\mathrm{N}}$ with $\frac{\mathrm{r}}{\mathrm{m}}$ and so the matrix element can be reduced to one of $r$. The electric dipole operator is independent of the momentum dependence of the Hamiltonian, the influence of the latter residing entirely in the initial and final wave functions employed in the evaluation of the matrix element. This is just Siegert's theorem. We therefore conclude that in the region in which (C.7) is valid we can obtain no direct evidence on the momentum dependence of the nucleon-nucleus interaction. For all other situations, namely those in which the space dependence of the electromagnetic potentials enters, the gauge substitution $\underline{p} \rightarrow \underset{m}{p}-\in A / c$ is generally not correct. It is valid only for electric dipole transitions. 


\section{ACKNOWLEDGENENTS}

I am indebted to Norman Francis and Carl Shakin for keeping me informed of their work on this subject. I am grateful to Torleif Ericson for criticizing this manuscript and for several suggestions. 
1) H. Feshbach, Ann, Phys. (N.Y.) 19, 287 (1962).

2) H. Feshbach, Ann. Phys. (N.Y.) 5, 357 (1958).

3) A.M. Lane and R.G. Thomas, Rev. Mod. Phys. 30, 257 (1958).

4) R.F. Christy and I. Duck, Nucl. Phys. 24, 89 (1961).

5) A.M. Lane, Nucl. Phys. 11, 625 (1959);

A.M. Lane and J.E. Lynn, Nucl. Phys. 11, 646 (1959).

6) N.C. Francis, D.T. Goldman and E. Guth, Phys. Rev. 120, 2175 (1960).

7) C. Shakin, Ann. Phys. (N.Y.), to be published.

8) N.C. Francis, private communication.

9) H. Feshbach and D.R. Yennie, Nucl. Phys. 37, 150 (1962).

10) See, for example, S.S. Schweber, Introduction to Relativistic Quantum Field Theory, p. 625, Row, Peterson and Co., Evanston, Ill., (1961).

11) F.E. Low, Phys. Rev. 110, 974 (1958). 